\title{
SLOWLY GROWING SOLUTIONS OF ODES REVISITED
}

\author{
Janne Gröhn \\ University of Eastern Finland, Department of Physics and Mathematics \\ P.O. Box 111, FI-80101 Joensuu, Finland; janne.grohn@uef.fi
}

\begin{abstract}
Solutions of the differential equation $f^{\prime \prime}+A f=0$ are considered assuming that $A$ is analytic in the unit disc $\mathbf{D}$ and satisfies

$$
\sup _{z \in \mathbf{D}}|A(z)|\left(1-|z|^{2}\right)^{2} \log \frac{e}{1-|z|}<\infty .
$$

By recent results in the literature, such restriction has been associated to coefficient conditions which place all solutions in the Bloch space $\mathcal{B}$. In this paper it is shown that any coefficient condition implying $(\star)$ fails to detect certain cases when Bloch solutions do appear. The converse problem is also addressed: What can be said about the growth of the coefficient $A$ if all solutions of $f^{\prime \prime}+A f=0$ belong to $\mathcal{B}$ ? An overall revised look into slowly growing solutions is presented, emphasizing function spaces $\mathcal{B}$, BMOA, VMOA.
\end{abstract}

\section{Introduction}

Let $\mathcal{H}(\mathbf{D})$ denote the collection of analytic functions in the (open) unit disc $\mathbf{D}$ of the complex plane $\mathbf{C}$. It is well-known that the growth of the coefficient $A \in \mathcal{H}(\mathbf{D})$ controls the growth of solutions $f \in \mathcal{H}(\mathbf{D})$ of the linear differential equation

$$
f^{\prime \prime}+A f=0,
$$

and vice versa. The recent study [7] concerns conditions, given in terms of the coefficient $A$, which imply that all solutions of (1) belong to a given space of slowly growing analytic functions. Special attention is paid to $\mathcal{B}$ (Bloch space), BMOA (analytic functions of bounded mean oscillation) and VMOA (analytic functions of vanishing mean oscillation). These coefficient conditions have in common that they all imply

$$
\|A\|_{\mathcal{L}^{1}}=\sup _{z \in \mathbf{D}}|A(z)|\left(1-|z|^{2}\right)^{2} \log \frac{e}{1-|z|}<\infty,
$$

which is the subject of this research. The operator theoretic approach in [7] is based on duality relations, in contrast to this paper, where more classical tools are employed.

The search for coefficient conditions forcing all solutions of (1) to be of slow growth has been active for many years. In the 1997 summer school Function Spaces and Complex Analysis (Mekrijärvi Research Station, Finland), Danikas posed the following problem:

(Q) Find a sharp condition for the coefficient $A$ which implies that all solutions of (1) belong to $\mathcal{B}$.

https://doi.org/10.5186/aasfm.2018.4339

2010 Mathematics Subject Classification: Primary 34C10; Secondary 30D45.

Key words: Bloch space, BMOA, growth of solution, linear differential equation, oscillation of solution, VMOA.

The author is supported in part by the Academy of Finland \#286877. 
It is known that, if $\|A\|_{\mathcal{L}^{1}}$ is sufficiently small, then all solutions of (1) belong to $\mathcal{B}$. This result was recently discovered with the best possible upper bound for $\|A\|_{\mathcal{L}^{1}}$ in [14, Corollary 4(b) and Example 5(b)]. This means that in the language of $\mathcal{L}^{1}$-norms, the problem $(\mathrm{Q})$ has been solved. The alternative approach in [7] produces a family of coefficient conditions, which all fall into the category $A \in \mathcal{L}^{1}$, see $[7$, Theorems 10 and 11].

Our intention is to take a revised look into slowly growing solutions of (1), and in particular, to concentrate to the borderline case $A \in \mathcal{L}^{1}$. We show that any coefficient condition implying $A \in \mathcal{L}^{1}$ is not sufficiently delicate to detect certain special cases when Bloch solutions do appear. In this sense, the problem (Q) remains open as the most natural description is yet to be found. The converse problem is addressed in Section 2.4 .

\section{Results}

2.1. Growth of solutions. Our first result solves the problem (Q) in terms of the maximum modulus $M_{\infty}(r, A)=\max _{|z|=r}|A(z)|, 0 \leq r<1$.

Theorem 1. Let $A \in \mathcal{H}(\mathbf{D})$. If there exists $0 \leq r_{0}<1$ such that

$$
\sup _{r_{0}<r<1} M_{\infty}(r, A)(1-r)^{2} \exp \left(\int_{r_{0}}^{r} M_{\infty}(t, A)(1-t) d t\right)<\infty
$$

then all solutions of (1) belong to $\mathcal{B}$.

Theorem 1 is based on a representation formula for solutions of (1) and the following elementary observation. If $f$ is a solution of (1) for $A \in \mathcal{H}(\mathbf{D})$, then $f$ belongs to the Bloch space

$$
\mathcal{B}=\left\{f \in \mathcal{H}(\mathbf{D}):\|f\|_{\mathcal{B}}=\sup _{z \in \mathbf{D}}\left|f^{\prime}(z)\right|\left(1-|z|^{2}\right)<\infty\right\}
$$

if and only if

$$
\sup _{z \in \mathbf{D}}|f(z)||A(z)|\left(1-|z|^{2}\right)^{2}<\infty .
$$

Theorem 1 sharpens [14, Corollary 4(b)], but fails to be an optimal solution to the problem (Q) as it shares the same defects with other known solutions; see Remarks 1 and 2 below.

The growth space $\mathcal{L}^{\alpha}$ for $0 \leq \alpha<\infty$ consists of those $A \in \mathcal{H}(\mathbf{D})$ for which

$$
\|A\|_{\mathcal{L}^{\alpha}}=\sup _{z \in \mathbf{D}}|A(z)|\left(1-|z|^{2}\right)^{2}\left(\log \frac{e}{1-|z|}\right)^{\alpha}<\infty .
$$

The space $\mathcal{L}^{0}$ appears several times in the literature, and is usually denoted by $H_{2}^{\infty}$ or $\mathcal{A}^{-2}$. In the sense of (3) it seems to be the correct ballpark for the study of Bloch solutions of (1). However, even if $\|A\|_{\mathcal{L}^{0}}$ is arbitrarily small, it is possible that all non-trivial solutions $(f \not \equiv 0)$ of (1) lie outside $\mathcal{B}$; see Example 2 below. If $A \in \mathcal{L}^{\alpha}$ for $1<\alpha<\infty$, then all solutions of (1) are bounded in $\mathbf{D}$ by [11, Theorem 4.2]. As explained in the Introduction, if $\|A\|_{\mathcal{L}^{1}}$ is sufficiently small, then all solutions of (1) belong to the Bloch space, while the weaker condition $A \in \mathcal{L}^{1}$ allows some solutions to lie outside $\mathcal{B}$. The following result is in line with the heuristic principle which claims that small change in $\|A\|_{\mathcal{L}^{1}}$ has a huge impact on solutions of (1).

Theorem 2. If $\|A\|_{\mathcal{L}^{1}}<4 / n$ for $n \in \mathbf{N}$, then all solutions $f$ of (1) satisfy $f, f^{2}, \ldots, f^{n} \in \mathcal{B}$. 
For $1 / 2<\alpha<\infty$, the coefficient condition $A \in \mathcal{L}^{\alpha}$ places all solutions of (1) in $\bigcap_{0<p<\infty} H^{p}$, see [20, Corollary 1.9]. This property is no longer true for $\alpha=1 / 2$ as certain solutions may lie outside the Nevanlinna class $\mathcal{N}$; apply [18, Theorem 4] to $Q(r)=(1-r)^{-2}(\log (e /(1-r)))^{-1 / 2}, 0 \leq r<1$. It seems that non-Nevanlinna solutions produced in this manner do not belong to $\mathcal{B}$ as they are exponentials of very badly behaved Bloch functions themselves. The following result indicates that not all Bloch solutions of (1) are smooth enough to be contained in $\mathcal{N}$. By the discussion above, these solutions cannot be detected by any coefficient condition which implies $A \in \mathcal{L}^{1}$.

As usual, the Hardy space $H^{p}$ for $0<p<\infty$ consists of $f \in \mathcal{H}(\mathbf{D})$ for which

$$
\|f\|_{H^{p}}^{p}=\lim _{r \rightarrow 1^{-}} \frac{1}{2 \pi} \int_{0}^{2 \pi}\left|f\left(r e^{i \theta}\right)\right|^{p} d \theta<\infty,
$$

while the Nevanlinna class $\mathcal{N}$ contains functions $f \in \mathcal{H}(\mathbf{D})$ such that

$$
\lim _{r \rightarrow 1^{-}} \frac{1}{2 \pi} \int_{0}^{2 \pi} \log ^{+}\left|f\left(r e^{i \theta}\right)\right| d \theta<\infty, \quad \log ^{+}=\max \{\log , 0\} .
$$

Here $\log ^{+} 0=0$ by definition.

Theorem 3. Let $0<C<\infty$. Then, there exists a coefficient $A \in \mathcal{H}(\mathbf{D})$ with $\|A\|_{\mathcal{L}^{0}}<C$ such that $(1)$ admits a (zero-free) solution $f \in \mathcal{B} \backslash \mathcal{N}$.

The following result complements Theorem 3 by offering a condition under which non-Nevanlinna solutions do not appear.

Theorem 4. If $\|A\|_{\mathcal{L}^{0}} \leq 1$ and there exists a zero-free solution of (1) which belongs to $\bigcup_{0<p<\infty} H^{p}$, then all solutions of (1) are in $\bigcup_{0<p<\infty} H^{p}$.

The coefficient condition $\|A\|_{\mathcal{L}^{0}} \leq 1$ corresponds to the classical univalency criterion [16, Theorem I] due to Nehari, which implies that all non-trivial solutions of (1) have at most one zero in $\mathbf{D}$. Theorem 4 should be compared to [10, Theorem 4] which holds in a more general setting.

2.2. Oscillation of solutions. If $A \in \mathcal{H}(\mathbf{D})$ and there exists $0<R<1$ such that $|A(z)|\left(1-|z|^{2}\right)^{2} \leq 1$ for all $R<|z|<1$, then all non-trivial solutions of (1) vanish at most finitely many times in $\mathbf{D}[23$, Theorem 1]. This is the case, in particular, if $A \in \mathcal{L}^{\alpha}$ for any $0<\alpha<\infty$. The following example concerns the situation when all solutions belong to $\mathcal{B}$ while one of them has infinitely many zeros. This is Hille's example, see [13] and [23, p. 162].

Example 1. Let $0<\gamma<\infty$. On one hand, all solutions of the differential equation (1) for $A(z)=\left(1+4 \gamma^{2}\right) /\left(1-z^{2}\right)^{2}, z \in \mathbf{D}$, are bounded and hence in $\mathcal{B}$. This follows from the estimates in [24, p. 131], for example. On the other hand, the particular solution

$$
f(z)=\sqrt{1-z^{2}} \sin \left(\gamma \log \frac{1+z}{1-z}\right), \quad z \in \mathbf{D},
$$

has infinitely many (real) zeros $z_{n}=\left(e^{\pi n / \gamma}-1\right) /\left(e^{\pi n / \gamma}+1\right), n \in \mathbf{Z}$.

Remark 1. By the discussion above, the coefficient condition $A \in \mathcal{L}^{1}$ implies that all non-trivial solutions of (1) belong to $\bigcap_{0<p<\infty} H^{p}$ and have at most finitely many zeros. We have shown that neither of these properties is characteristic to Bloch solutions of (1) under the restriction $A \in \mathcal{L}^{0}$. 
We point out that, although $A \in \mathcal{L}^{1}$ is not sufficient to place all solutions of (1) in $\mathcal{B}$, it guarantees that solutions are normal in the sense

$$
\sup _{z \in \mathbf{D}} f^{\#}(z)\left(1-|z|^{2}\right)=\sup _{z \in \mathbf{D}} \frac{\left|f^{\prime}(z)\right|}{1+|f(z)|^{2}}\left(1-|z|^{2}\right)<\infty .
$$

This follows from [8, Proposition 7] by using the fact that all non-trivial solutions have at most finitely many zeros provided that $A \in \mathcal{L}^{1}$.

2.3. Solutions of finite valance. Let $n(f, \zeta)=\#\{z \in \mathbf{D}: f(z)=\zeta\}$ be the counting function for $\zeta$-points of $f \in \mathcal{H}(\mathbf{D})$; let $D(z, r)$ denote the Euclidean disc of radius $0<r<\infty$ centered at $z \in \mathbf{D}$; and let $d m$ be the Lebesgue area measure. According to [19, Satz 1], if $f \in \mathcal{B}$ and

$$
V_{f}=\sup _{z \in \mathbf{C}} \int_{D(z, 1)} n(f, \zeta) d m(\zeta)<\infty,
$$

then $f \in$ BMOA. Hence, Bloch functions of finite valence belong to BMOA. Recall that $f \in \mathrm{BMOA}$ if and only if $\|f\|_{\mathrm{BMOA}}^{2}=\sup _{a \in \mathbf{D}}\left\|g_{a}\right\|_{H^{2}}^{2}<\infty$, where $g_{a}(z)=$ $f\left(\varphi_{a}(z)\right)-f(a)$ and $\varphi_{a}(z)=(a-z) /(1-\bar{a} z)$ for $a, z \in \mathbf{D}$.

If $\|A\|_{\mathcal{L}^{1}}$ is sufficiently small, then all finitely valent solutions of (1) are not only in BMOA but also possess a specific type of regularity.

Theorem 5. Let $A \in \mathcal{L}^{1}$, and let $\beta$ be any constant such that $\|A\|_{\mathcal{L}^{1}} / 2<\beta<\infty$. If $f$ is a solution of (1) which satisfies (4), then

$$
\int_{\mathbf{D}}\left|f^{\prime}(z)\right|^{2}\left(\log \frac{e}{1-|z|}\right)^{-\beta} d m(z)<\infty .
$$

Example 3 below shows that, regardless of the size of $\|A\|_{\mathcal{L}^{1}}$, both finitely and infinitely valent (non-trivial) solutions of (1) are possible.

2.4. Converse problem. Before going any further, we discuss a problem converse to Theorem 1: How is the growth of the coefficient $A \in \mathcal{H}(\mathbf{D})$ restricted if all solutions of $(1)$ are in $\mathcal{B}$ ?

The argument in [24] reveals the following estimates. Let $f_{1}, f_{2}$ be linearly independent bounded solutions of (1) for $A \in \mathcal{H}(\mathbf{D})$. Without any loss of generality, we may assume that $f_{1} f_{2}^{\prime}-f_{1}^{\prime} f_{2}=1$. By a straight-forward computation $A=f_{1}^{\prime} f_{2}^{\prime \prime}-f_{1}^{\prime \prime} f_{2}^{\prime}$, and therefore $\sup _{z \in \mathbf{D}}|A(z)|\left(1-|z|^{2}\right)^{3}<\infty$. Moreover, the spherical derivative $w^{\#}=\left|w^{\prime}\right| /\left(1+|w|^{2}\right)$ of $w=f_{1} / f_{2}$ satisfies $w^{\#}=1 /\left(\left|f_{1}\right|^{2}+\left|f_{2}\right|^{2}\right) \leq\left|f_{1}^{\prime}\right|^{2}+\left|f_{2}^{\prime}\right|^{2}$, and hence $\sup _{z \in \mathbf{D}} w^{\#}(z)\left(1-|z|^{2}\right)^{2}<\infty$. It is clear that these estimates withstand the weaker assumption $f_{1}, f_{2} \in \mathcal{B}$. The following result improves the growth estimate for $A$ and is related to a problem mentioned in [24, p. 131].

Theorem 6. Let $f_{1}, f_{2} \in \mathcal{B}$ be linearly independent solutions of (1) for $A \in$ $\mathcal{H}(\mathbf{D})$. Then, $\sup _{z \in \mathbf{D}}|A(z)|\left(1-|z|^{2}\right)^{5 / 2} \lesssim \max \left\{\left\|f_{1}\right\|_{\mathcal{B}},\left\|f_{2}\right\|_{\mathcal{B}}\right\}<\infty$.

Here $\lesssim$ denotes a one sided estimate up to a constant. The betting is that Theorem 6 is not sharp. It would be desirable to show $A \in \mathcal{L}^{0}$ if $f_{1}, f_{2} \in \mathcal{B}$. We do not know whether this is true (even for $f_{1}, f_{2}$ bounded), however. Theorem 6 fails to be true if we have information only on one non-trivial solution of (1). For example, $f(z)=\exp (-(1+z) /(1-z))$ is a bounded solution of $(1)$ for $A(z)=-4 z /(1-z)^{4}$, $z \in \mathbf{D}$. In this case (1) admits also non-Bloch solutions such as

$$
f(z) \int_{0}^{z} \frac{1}{f(\zeta)^{2}} d \zeta, \quad z \in \mathbf{D}
$$


which is linearly independent to $f$ and grows too fast on the positive real axis to be included in $\mathcal{B}$ (by the Bernoulli-l'Hôpital theorem).

Let $A \in \mathcal{H}(\mathbf{D})$. If there exist linearly independent bounded solutions $f_{1}, f_{2}$ of (1) such that $\inf _{z \in \mathbf{D}}\left(\left|f_{1}(z)\right|+\left|f_{2}(z)\right|\right)>0$, then $A \in \mathcal{L}^{0}$ by an argument based on the corona theorem [7, p. 3]. We extend this observation for $\mathcal{B}$ with an argument independent of the corona theorem.

Theorem 7. Let $f_{1}, f_{2} \in \mathcal{B}$ be linearly independent solutions of (1) for $A \in$ $\mathcal{H}(\mathbf{D})$ such that $\inf _{z \in \mathbf{D}}\left(\left|f_{1}(z)\right|+\left|f_{2}(z)\right|\right)>0$. Then, $A \in \mathcal{L}^{0}$ and $\left(f_{1} / f_{2}\right)^{\#}$ is bounded in $\mathrm{D}$.

2.5. Solutions of bounded and vanishing mean oscillation. Coefficient conditions, which place all solutions of (1) in BMOA, are considered in [7]. We derive a result similar to [7, Theorem 3] by using known growth estimates for solutions of (1). This method is somewhat surprising, since it was not known to work with slowly growing solutions. By the Carleson measure description in [26, Theorem 1], Theorem 8 is weaker than [7, Theorem 3].

Theorem 8. Let $A \in \mathcal{H}(\mathbf{D})$. If

$$
\sup _{a \in \mathbf{D}}\left(\log \frac{e}{1-|a|}\right) \int_{\mathbf{D}}|A(z)|\left(1-\left|\varphi_{a}(z)\right|^{2}\right) d m(z)
$$

is sufficiently small, then all solutions of (1) belong to BMOA.

Coefficient conditions, which place all solutions of (1) in VMOA, are also discussed in [7]. We consider two related results which, as opposed to ones in [7], are given in terms of the radial growth of the coefficient. Recall that $f \in \mathrm{VMOA}$ if and only if $\left\|g_{a}\right\|_{H^{2}}^{2} \rightarrow 0^{+}$as $|a| \rightarrow 1^{-}$, where $g_{a}$ is as above.

Theorem 9. Let $A \in \mathcal{H}(\mathbf{D})$. If there exists $0 \leq r_{0}<1$ such that

$$
\int_{r_{0}}^{1} M_{\infty}(r, A)^{2} \exp \left(2 \int_{r_{0}}^{r} M_{\infty}(t, A)(1-t) d t\right)\left(1-r^{2}\right)^{3} d r<\infty,
$$

then all solutions of (1) belong to VMOA.

Theorem 9 gives rise to the following corollary. The coefficient condition (8) allows solutions of (1) to be unbounded; see Example 4 in Section 10.

Corollary 10. Let $A \in \mathcal{H}(\mathbf{D})$. If

$$
\sup _{z \in \mathbf{D}}|A(z)|\left(1-|z|^{2}\right)^{2}\left(\log \frac{e}{1-|z|}\right) \log \log \frac{e}{1-|z|}<\infty
$$

then all solutions of (1) belong to VMOA.

\section{Proof of Theorem 1}

The following proof is based on the growth estimate [11, Theorem 4.2] for solutions of (1). The known approaches to Bloch solutions of (1) depend on other methods (duality relations [7] and straight-forward integration [14]).

Proof of Theorem 1. Let $f$ be a non-trivial solution of (1), and let $0 \leq r_{0}<1$ be fixed. If $r_{0}<r<1$ and $e^{i \theta} \in \partial \mathbf{D}$, then

$$
f\left(r e^{i \theta}\right)=f\left(r_{0} e^{i \theta}\right)+f^{\prime}\left(r_{0} e^{i \theta}\right)\left(r e^{i \theta}-r_{0} e^{i \theta}\right)-\int_{r_{0} e^{i \theta}}^{r e^{i \theta}} f(\zeta) A(\zeta)\left(r e^{i \theta}-\zeta\right) d \zeta,
$$


by the representation theorem [11, Theorem 4.1]. Therefore

$$
\left|f\left(r e^{i \theta}\right)\right| \leq\left(M_{\infty}\left(r_{0}, f\right)+M_{\infty}\left(r_{0}, f^{\prime}\right)\left(1-r_{0}\right)\right) \exp \left(\int_{r_{0}}^{r}\left|A\left(t e^{i \theta}\right)\right|(1-t) d t\right)
$$

by Gronwall's lemma [15, Lemma 5.10]. This growth estimate, the assumption (2), and the identity $f^{\prime \prime}=-A f$ imply that $f^{\prime \prime} \in \mathcal{L}^{0}$. This completes the proof as $f \in \mathcal{B}$ by [27, Theorem 5.4].

We proceed to show that Theorem 1 sharpens [14, Corollary 4(b)].

Remark 2. Suppose that the coefficient condition in [14, Corollary 4(b)] holds, that is, $A \in \mathcal{H}(\mathbf{D})$ and

$$
\sup _{z \in \mathbf{D}}|A(z)|(1-|z|)^{2} \int_{0}^{|z|} \frac{d r}{1-r}<1 .
$$

Fix any $0<r_{0}<1$, and compute

$$
\begin{aligned}
& \sup _{r_{0}<r<1} M_{\infty}(r, A)(1-r)^{2} \exp \left(\int_{r_{0}}^{r} M_{\infty}(t, A)(1-t) d t\right) \\
& \leq \sup _{r_{0}<r<1} \frac{1}{\log \frac{1}{1-r}} \exp \left(\log \log \frac{1}{1-r}-\log \log \frac{1}{1-r_{0}}\right)=\frac{1}{\log \frac{1}{1-r_{0}}} .
\end{aligned}
$$

Therefore, the assumptions of Theorem 1 are satisfied. We point out that Theorem 1 applies also to cases such as $A(z)=(1-z)^{-2}(\log (e /(1-z)))^{-1}, z \in \mathbf{D}$, for which [14, Corollary 4(b)] is inconclusive. In particular, Theorem 1 can be utilized even with the equality in (9).

Under an additional smoothness assumption, the coefficient condition (2) falls also into the category $A \in \mathcal{L}^{1}$. This is the case, for example, if the function $M_{\infty}(r, A)(1-r)^{2}\left(\log (e /(1-r)), r_{0}<r<1\right.$, is increasing.

The following example shows that, even if $\|A\|_{\mathcal{L}^{0}}$ is arbitrarily small, it is possible that all non-trivial solutions of (1) lie outside $\mathcal{B}$.

Example 2. Let $1<\gamma<\infty$ be fixed. The differential equation (1) for $A(z)=$ $\left(1-\gamma^{2}\right) /\left(1-z^{2}\right)^{2}, z \in \mathbf{D}$, admits linearly independent solutions

$$
f_{1}(z)=\frac{(1+z)^{(\gamma+1) / 2}}{(1-z)^{(\gamma-1) / 2}}, \quad f_{2}(z)=\frac{(1-z)^{(\gamma+1) / 2}}{(1+z)^{(\gamma-1) / 2}}, \quad z \in \mathbf{D},
$$

which clearly satisfy $f_{1}, f_{2} \notin \mathcal{B}$. Since the singularities of $f_{1}, f_{2}$ are located at distinct points, we conclude that all linear combinations of $f_{1}, f_{2}$, and therefore all non-trivial solutions of (1), lie outside $\mathcal{B}$.

\section{Proof of Theorem 2}

We begin with an auxiliary result, which shows that the coefficient condition $A \in \mathcal{L}^{1}$ is associated with solutions of at most logarithmic growth. This should be compared to the case of the coefficient condition $A \in \mathcal{L}^{0}$, which implies that all solutions of (1) satisfy $\sup _{z \in \mathbf{D}}|f(z)|\left(1-|z|^{2}\right)^{p}<\infty$ for sufficiently large $p=$ $p\left(\|A\|_{\mathcal{L}^{0}}\right)<\infty$, see [18, Example 1]. 
Lemma 11. Let $A \in \mathcal{L}^{1}$.

(i) If $\alpha$ is any constant such that $\|A\|_{\mathcal{L}^{1}} / 4<\alpha<\infty$, then all solutions $f$ of (1) satisfy

$$
\sup _{z \in \mathbf{D}}|f(z)|\left(\log \frac{e}{1-|z|}\right)^{-\alpha}<\infty .
$$

(ii) Any solution $f$ of (1), which satisfies (10) for $\alpha=1$, belongs to $\mathcal{B}$.

The proof of Lemma 11(i) resembles that of [9, Theorem 2]; a similar estimate could also be obtained from [11, Theorem 4.2]. Lemma 11(ii) is an immediate consequence of (3) and [27, Theorem 5.4], but plays an important role in the proof of Theorem 2.

Proof of Lemma 11. (i) Let $f$ be a solution of (1), and $0 \leq \delta<R<1$. Since

$$
|f(z)| \leq \int_{\delta}^{|z|} \int_{\delta}^{t}\left|f^{\prime \prime}(s z /|z|)\right| d s d t+M_{\infty}\left(\delta, f^{\prime}\right)+M_{\infty}(\delta, f), \quad \delta<|z|<1,
$$

we obtain

$$
\begin{aligned}
\sup _{\delta<|z|<R} \frac{|f(z)|}{\left(\log \frac{e}{1-|z|}\right)^{\alpha}} \leq & \left(\sup _{\delta<|\zeta|<R} \frac{|f(\zeta)|}{\left(\log \frac{e}{1-|\zeta|}\right)^{\alpha}}\right)\|A\|_{\mathcal{L}^{1}} \sup _{\delta<|z|<R} I_{\alpha}(z) \\
& +M_{\infty}\left(\delta, f^{\prime}\right)+M_{\infty}(\delta, f),
\end{aligned}
$$

where $I_{\alpha}(z)$ is as below. Since

$$
\lim _{|z| \rightarrow 1^{-}} I_{\alpha}(z)=\lim _{|z| \rightarrow 1^{-}}\left(\log \frac{e}{1-|z|}\right)^{-\alpha} \int_{0}^{|z|} \int_{0}^{t} \frac{\left(\log \frac{e}{1-s}\right)^{\alpha-1}}{\left(1-s^{2}\right)^{2}} d s d t=\frac{1}{4 \alpha}
$$

by the Bernoulli-l'Hôpital theorem, we deduce (10) for $\|A\|_{\mathcal{L}^{1}} / 4<\alpha<\infty$ by choosing a sufficiently large $0 \leq \delta<1$, reorganizing the terms and finally letting $R \rightarrow 1^{-}$.

Proof of Theorem 2. Note that $f \in \mathcal{B}$ follows directly from Lemma 11; first, apply the part (i) and then (ii). If $n=1$ then there is nothing else to prove.

We proceed by induction. Assume that $f^{k-1} \in \mathcal{B}$, where the parameter $k$ satisfies $2 \leq k \leq n$. Since $\|A\|_{\mathcal{L}^{1}}<4 / k$ by the assumption, every solution $f$ of (1) satisfies (10) for $\alpha=1 / k$ according to Lemma 11(i). Now

$$
\left(f^{k}\right)^{\prime \prime}=k f^{\prime}\left(f^{k-1}\right)^{\prime}-k f^{k} A
$$

by (1). We deduce $\left(f^{k}\right)^{\prime \prime} \in \mathcal{L}^{0}$, which gives $f^{k} \in \mathcal{B}$. The claim follows.

\section{Proof of Theorem 3}

The following proof takes advantage of universal covering maps in creation of Bloch function with special properties. Similar arguments appear in the literature several times. The idea for the following Bloch construction is borrowed from $[3$, p. 229].

Proof of Theorem 3. Let $0<C<\infty$. By the proof of [18, Theorem 4], when applied to $Q(r)=C /(1-r)^{2}$, there exists $g \in \mathcal{B} \backslash \mathcal{N}$ with $\|g\|_{\mathcal{B}} \lesssim C$ such that $f=e^{g} \notin \mathcal{N}$ is a solution of (1) for $A=-g^{\prime \prime}-\left(g^{\prime}\right)^{2}$ with $\|A\|_{\mathcal{L}^{0}} \leq 4 C$.

Let $\mathcal{Z}=\{x+i y \in \mathbf{C}: x, y \in \mathbf{Z}\}$ be the set of integral lattice points, and let $E$ be its preimage $E=\{z \in \mathbf{D}: f(z) \in \mathcal{Z}\}$. Since $E \subset \mathbf{D}$ is a countable closed set, $E$ has (logarithmic) capacity zero and therefore the universal covering map from $\mathbf{D}$ 
onto $\mathbf{D} \backslash E$ is an inner function [6]; see also [25, p. 261]. Let this inner function be denoted by $I$. The function $f \circ I$ belongs to $\mathcal{B}$ since its image, contained in $\mathbf{C} \backslash \mathcal{Z}$, does not contain (schlicht) discs of arbitrarily large radius; see [4, Theorem 2.6], for example. Note that $f \circ I$ is non-vanishing, and define $B \in \mathcal{H}(\mathbf{D})$ by

$$
B=-\frac{(f \circ I)^{\prime \prime}}{f \circ I}=(A \circ I)\left(I^{\prime}\right)^{2}-\left(g^{\prime} \circ I\right) I^{\prime \prime}
$$

By the Schwarz-Pick lemma, and its extension [22, Theorem 2], we deduce

$$
\begin{aligned}
\|B\|_{\mathcal{L}^{0}} \leq & \sup _{z \in \mathbf{D}}\left(1-|z|^{2}\right)^{2} \frac{\|A\|_{\mathcal{L}^{0}}}{\left(1-|I(z)|^{2}\right)^{2}} \cdot \frac{\left(1-|I(z)|^{2}\right)^{2}}{\left(1-|z|^{2}\right)^{2}} \\
& +\sup _{z \in \mathbf{D}}\left(1-|z|^{2}\right)^{2} \frac{\|g\|_{\mathcal{B}}}{1-|I(z)|^{2}} \cdot \frac{2 !\left(1-|I(z)|^{2}\right)}{(1-|z|)^{2}(1+|z|)} \\
\leq & \|A\|_{\mathcal{L}^{0}}+4\|g\|_{\mathcal{B} .}
\end{aligned}
$$

We conclude that $h=f \circ I \in \mathcal{B}$ is a zero-free solution of $h^{\prime \prime}+B h=0$, where $\|B\|_{\mathcal{L}^{0}} \lesssim$ $C$ with a comparison constant independent of $C$. Finally, [25, Proposition 3.3] implies that $f \circ I$ does not belong to $\mathcal{N}$.

\section{Proof of Theorem 4}

The following result shows that slow growth of the coefficient ensures the existence of zero-free solution bases.

Lemma 12. If $\|A\|_{\mathcal{L}^{0}} \leq 1$, then (1) admits linearly independent zero-free solutions $f_{1}$ and $f_{2}$ such that $\log f_{1}-\log f_{2} \in$ BMOA.

If $A \in \mathcal{L}^{0}$, then any zero-free solution $f$ of (1) satisfies $\log f \in \mathcal{B}$ by [8, Theorem 4(ii)]. The contribution of Lemma 12 lies in the fact that linearly independent zero-free solutions are shown to be closely related to each other. If $A \in \mathcal{L}^{1}$, then any zero-free solution $f$ of (1) satisfies $\log f \in$ BMOA by [8, Theorem 4(i)], and therefore the $\mathcal{L}^{1}$-counterpart of Lemma 12 is trivial.

Proof of Lemma 12. Let $g_{1}$ and $g_{2}$ be linearly independent solutions of (1) where $\|A\|_{\mathcal{L}^{0}} \leq 1$. It follows that $h=g_{1} / g_{2}$ is a locally univalent meromorphic (not necessarily analytic) function whose Schwarzian derivative $S_{h}=2 \mathrm{~A}$ satisfies $\left\|S_{h}\right\|_{\mathcal{L}^{0}} \leq 2$; see [15, Theorem 6.1], for example. Therefore $h$ is univalent in $\mathbf{D}$ by [16, Theorem I]. Consequently, there exist two distinct values $\zeta_{1}, \zeta_{2} \in \mathbf{C} \cup\{\infty\}$ which belong to the complement of $h(\mathbf{D})$ with respect to the extended complex plane. If $\zeta_{j} \in \mathbf{C}$ then define $f_{j}=g_{1}-\zeta_{j} g_{2}$, while otherwise let $f_{j}=g_{2}$. We conclude that $f_{1}$ and $f_{2}$ are linearly independent zero-free solutions of (1).

Finally, $w=f_{1} / f_{2}$ is a locally univalent analytic zero-free function, whose Schwarzian derivative agrees with $S_{h}$. It follows that $w$ is univalent, and therefore $\log w \in$ BMOA by [2, Corollary 1, p. 21]. The claim follows.

Proof of Theorem 4. Let $f_{1}$ and $f_{2}$ be linearly independent non-vanishing solutions of (1). Their existence follows from $\|A\|_{\mathcal{L}^{0}} \leq 1$ as in the proof of Lemma 12. Without loss of generality, we may assume that $f_{2} \in \bigcup_{0<p<\infty} H^{p}$ is the zero-free solution given by the hypothesis. Any solution $f$ of (1) can be represented in the form $f=\alpha f_{1}+\beta f_{2}=f_{2}\left(\alpha e^{\log f_{1}-\log f_{2}}+\beta\right)$, where $\alpha, \beta \in \mathbf{C}$ are constants depending on $f$. Since $\log f_{1}-\log f_{2} \in$ BMOA by Lemma 12 , we deduce $\exp \left(\log f_{1}-\log f_{2}\right) \in$ $\bigcup_{0<p<\infty} H^{p}$ by [5, Theorem 1]. This proves the assertion. 


\section{Proof of Theorem 5}

Theorem 5 reveals that finitely valent solutions possess a unique property, which is not even found from all bounded analytic functions. To construct a bounded function $f \in \mathcal{H}(\mathbf{D})$ for which (5) fails, consider a Blaschke sequence which is not a zero-sequence for the weighted Dirichlet space $\mathcal{D}_{s}$ for fixed $0<s<1$, and let $f$ be the corresponding Blaschke product. See [17, p. 1981] for more details.

Proof of Theorem 5. Let $\beta$ be any constant such that $\|A\|_{\mathcal{L}^{1}} / 2<\beta<\infty$, and fix $\alpha$ such that $\|A\|_{\mathcal{L}^{1}} / 4<\alpha<\beta / 2$. Since $f$ is a solution of (1) for $A \in \mathcal{L}^{1}$, (10) holds by Lemma 11(i). As in [19, p. 593], we compute

$$
\begin{aligned}
\int_{D(0, r)}\left|f^{\prime}(z)\right|^{2} d m(z) & =\int_{f(D(0, r))}\left(\sum_{z \in \mathbf{D}: f(z)=\zeta} 1\right) d m(\zeta) \\
& \leq \int_{D\left(0, M_{\infty}(r, f)\right)} n(f, \zeta) d m(\zeta) \leq 4\left(M_{\infty}(r, f)+1\right)^{2} \cdot V_{f} \\
& \lesssim\left(\log \frac{e}{1-r}\right)^{2 \alpha}, \quad 0<r<1
\end{aligned}
$$

by (4) and the generic change of variable formula [1, Proposition 2.1]. Now

$$
\begin{aligned}
& \int_{\mathbf{D}}\left|f^{\prime}(z)\right|^{2}\left(\log \frac{e}{1-|z|}\right)^{-\beta} d m(z) \\
& =\int_{0}^{1}\left(\int_{D(0, r)}\left|f^{\prime}(z)\right|^{2} d m(z)\right) \frac{\beta}{(1-r)\left(\log \frac{e}{1-r}\right)^{\beta+1}} d r \\
& \lesssim \int_{0}^{1} \frac{d r}{(1-r)\left(\log \frac{e}{1-r}\right)^{1+\beta-2 \alpha}}<\infty
\end{aligned}
$$

by Fubini's theorem.

The following example concerns the valence of solutions of (1).

Example 3. Let $0<\alpha<1$. As in [14, Example 5(b)], we conclude that $f(z)=(\log (e /(1-z)))^{\alpha}$ is a solution of (1) for

$$
A(z)=\frac{-\alpha}{(1-z)^{2}}\left((\alpha-1)\left(\log \frac{e}{1-z}\right)^{-2}+\left(\log \frac{e}{1-z}\right)^{-1}\right), \quad z \in \mathbf{D},
$$

where $\|A\|_{\mathcal{L}^{1}} \lesssim \alpha$. Since $z \mapsto \log (e /(1-z))$ is univalent in $\mathbf{D}$, we see that $f$ is finitely valent for $\alpha \in(0,1) \cap \mathbf{Q}$ and infinitely valent for $\alpha \in(0,1) \backslash \mathbf{Q}$.

\section{Proofs of Theorems 6 and 7}

Let $f_{1}, f_{2}$ be linearly independent solutions of (1) for $A \in \mathcal{H}(\mathbf{D})$. We may assume that the Wronskian determinant satisfies $f_{1} f_{2}^{\prime}-f_{1}^{\prime} f_{2}=1$. Differentiate this identity once to obtain $f_{1} f_{2}^{\prime \prime}-f_{1}^{\prime \prime} f_{2}=0$, and differentiate it twice to deduce $f_{1}^{\prime \prime \prime} f_{2}-f_{1} f_{2}^{\prime \prime \prime}=f_{1}^{\prime} f_{2}^{\prime \prime}-f_{1}^{\prime \prime} f_{2}^{\prime}=A$, where the last equality follows from (1).

Proof of Theorem 6. Since $f_{1}, f_{2} \in \mathcal{B}$, we conclude that $f_{1}^{\prime \prime}, f_{2}^{\prime \prime} \in \mathcal{L}^{0}$. Define $h(z)=\left|f_{1}(z)\right|+\left|f_{2}(z)\right|, z \in \mathbf{D}$. Function $h$ is non-vanishing as the Wronskian 
determinant satisfies $f_{1} f_{2}^{\prime}-f_{1}^{\prime} f_{2}=1$. On one hand,

$$
\begin{aligned}
|A(z)| & =\frac{\left|f_{1}(z) A(z)\right|+\left|f_{2}(z) A(z)\right|}{\left|f_{1}(z)\right|+\left|f_{2}(z)\right|}=\frac{\left|f_{1}^{\prime \prime}(z)\right|+\left|f_{2}^{\prime \prime}(z)\right|}{\left|f_{1}(z)\right|+\left|f_{2}(z)\right|} \\
& \lesssim \frac{\max \left\{\left\|f_{1}\right\|_{\mathcal{B}},\left\|f_{2}\right\|_{\mathcal{B}}\right\}}{\left(1-|z|^{2}\right)^{3}} \cdot \frac{1-|z|^{2}}{h(z)}, \quad z \in \mathbf{D},
\end{aligned}
$$

with an absolute comparison constant. On the other hand,

$$
|A(z)| \leq\left|f_{1}^{\prime \prime \prime}(z)\right|\left|f_{2}(z)\right|+\left|f_{1}(z)\right|\left|f_{2}^{\prime \prime \prime}(z)\right| \lesssim \frac{\max \left\{\left\|f_{1}\right\|_{\mathcal{B}},\left\|f_{2}\right\|_{\mathcal{B}}\right\}}{\left(1-|z|^{2}\right)^{3}} h(z), \quad z \in \mathbf{D} .
$$

Since $\min \{x / y, y\} \leq \sqrt{x}$ for all $0<x, y<\infty$, we obtain

$$
\min \left\{\left(1-|z|^{2}\right) / h(z), h(z)\right\} \leq \sqrt{1-|z|^{2}}, \quad z \in \mathbf{D} .
$$

The assertion $\sup _{z \in \mathbf{D}}|A(z)|\left(1-|z|^{2}\right)^{5 / 2} \lesssim \max \left\{\left\|f_{1}\right\|_{\mathcal{B}},\left\|f_{2}\right\|_{\mathcal{B}}\right\}$ follows.

The proof of Theorem 7 is similar to the one above, with the difference that the auxiliary function $h$ in the proof of Theorem 6 , is now uniformly bounded away from zero by the assumption.

Proof of Theorem 7. Since $f_{1}, f_{2} \in \mathcal{B}$, we have $f_{1}^{\prime \prime}, f_{2}^{\prime \prime} \in \mathcal{L}^{0}$. By (1),

$$
\begin{aligned}
\sup _{z \in \mathbf{D}}|A(z)|\left(1-|z|^{2}\right)^{2} & =\sup _{z \in \mathbf{D}} \frac{\left|f_{1}(z) A(z)\right|+\left|f_{2}(z) A(z)\right|}{\left|f_{1}(z)\right|+\left|f_{2}(z)\right|}\left(1-|z|^{2}\right)^{2} \\
& \leq\left(\inf _{z \in \mathbf{D}}\left(\left|f_{1}(z)\right|+\left|f_{2}(z)\right|\right)\right)^{-1}\left(\left\|f_{1}^{\prime \prime}\right\|_{\mathcal{L}^{0}}+\left\|f_{2}^{\prime \prime}\right\|_{\mathcal{L}^{0}}\right) .
\end{aligned}
$$

Let $w=f_{1} / f_{2}$, which implies that $w^{\prime}=-1 / f_{2}^{2}$. To see that $w^{\#}$ is bounded in $\mathbf{D}$, it suffices to write

$$
\begin{aligned}
\sup _{z \in \mathbf{D}} w^{\#}(z) & =\sup _{z \in \mathbf{D}} \frac{1}{\left|f_{1}(z)\right|^{2}+\left|f_{2}(z)\right|^{2}} \leq \sup _{z \in \mathbf{D}} \frac{2}{\left(\left|f_{1}(z)\right|+\left|f_{2}(z)\right|\right)^{2}} \\
& \leq 2\left(\inf _{z \in \mathbf{D}}\left(\left|f_{1}(z)\right|+\left|f_{2}(z)\right|\right)\right)^{-2} .
\end{aligned}
$$

This completes the proof.

We take the opportunity to mention an interesting application of (11). Let $f_{1}, f_{2}$ be linearly independent solutions of (1) for $A \in \mathcal{H}(\mathbf{D})$ such that $\inf _{z \in \mathbf{D}}\left(\left|f_{1}(z)\right|+\right.$ $\left.\left|f_{2}(z)\right|\right)>0$, and let $z_{1}, z_{2} \in \mathbf{D}$ be (necessarily distinct) points at which $f_{1}\left(z_{1}\right)=$ $0=f_{2}\left(z_{2}\right)$. Let $\gamma\left(z_{1}, z_{2}\right)$ denote the straight line segment from $z_{1}$ to $z_{2}$. Since $z_{1}$ is a (simple) zero of $w=f_{1} / f_{2}$, and $z_{2}$ is a (simple) pole of $w$, we deduce

$$
1 \lesssim \int_{\gamma\left(z_{1}, z_{2}\right)} \frac{\left|w^{\prime}(z)\right|}{1+|w(z)|^{2}}|d z| \leq\left(\sup _{z \in \mathbf{D}} w^{\#}(z)\right)\left|z_{1}-z_{2}\right|
$$

as the spherical length of $w\left(\gamma\left(z_{1}, z_{2}\right)\right)$ is uniformly bounded from below. Therefore, (11) implies that $\left|z_{1}-z_{2}\right|$ is uniformly bounded away from zero.

\section{Proof of Theorem 8}

By [26, Theorem 1] and the subharmonicity of $|A|$, we deduce

$$
\|A\|_{\mathcal{L}^{1}} \lesssim \sup _{a \in \mathbf{D}}\left(\log \frac{e}{1-|a|}\right) \int_{\mathbf{D}}|A(z)|\left(1-\left|\varphi_{a}(z)\right|^{2}\right) d m(z)
$$


Consequently, when proving Theorem 8, we may assume that all solutions of (1) are in $\mathcal{B}$ by $[14$, Corollary $4(\mathrm{~b})]$ or Theorem 1.

Proof of Theorem 8. Let $f$ be a solution of (1) and consider its normalized hyperbolic translates $g_{a}(z)=f\left(\varphi_{a}(z)\right)-f(a)$ for $a \in \mathbf{D}$. To prove $f \in$ BMOA it suffices to show that

$$
\sup _{a \in \mathbf{D}} \sup _{0<r<1} m\left(r, g_{a}\right)=\sup _{a \in \mathbf{D}} \sup _{0<r<1} \frac{1}{2 \pi} \int_{0}^{2 \pi} \log ^{+}\left|g_{a}\left(r e^{i \theta}\right)\right| d \theta<\infty
$$

by $[2$, Corollary 2, p. 15$]$. We proceed to verify that the proximity functions $m\left(r, g_{a}\right)$ satisfy (12).

A straight-forward computation reveals that $g_{a} \in \mathcal{H}(\mathbf{D})$ is a solution of the nonhomogenous linear differential equation

$$
g_{a}^{\prime \prime}+B_{a} g_{a}^{\prime}+C_{a} g_{a}=-f(a) C_{a},
$$

where $B_{a}, C_{a} \in \mathcal{H}(\mathbf{D})$ are given by

$$
B_{a}(z)=-\frac{\varphi_{a}^{\prime \prime}(z)}{\varphi_{a}^{\prime}(z)}, \quad C_{a}(z)=A\left(\varphi_{a}(z)\right) \varphi_{a}^{\prime}(z)^{2}, \quad z \in \mathbf{D}
$$

By [12, Corollary 3(a)] and the proof of [12, Theorem 1(a)], we deduce

$$
\begin{aligned}
m\left(r, g_{a}\right) \lesssim 1 & +\log ^{+}\left(\left|f^{\prime}(a)\right|\left(1-|a|^{2}\right)\right) \\
& +\int_{0}^{2 \pi} \log ^{+}\left(\int_{0}^{r}\left|A\left(\varphi_{a}\left(s e^{i \theta}\right)\right)\right|\left|\varphi_{a}^{\prime}\left(s e^{i \theta}\right)\right|^{2}|f(a)|(1-s) d s\right) d \theta \\
& +\int_{D(0, r)}\left|A\left(\varphi_{a}(z)\right)\right|\left|\varphi_{a}^{\prime}(z)\right|^{2}\left(1-|z|^{2}\right) d m(z) \\
& +\int_{D(0, r)}\left|\frac{\varphi_{a}^{\prime \prime}(z)}{\varphi_{a}^{\prime}(z)}\right| d m(z)+\int_{D(0, r)}\left|\left(\frac{\varphi_{a}^{\prime \prime}}{\varphi_{a}^{\prime}}\right)^{\prime}(z)\right|\left(1-|z|^{2}\right) d m(z)
\end{aligned}
$$

for all $0<r<1$, where the comparison constant is independent of $a \in \mathbf{D}$. The area integrals involving $B_{a}$ and $B_{a}^{\prime}$ are uniformly bounded for $0<r<1$ and $a \in \mathbf{D}$ by standard estimates, while

$$
\sup _{a \in \mathbf{D}} \sup _{0<r<1} \int_{D(0, r)}\left|A\left(\varphi_{a}(z)\right)\right|\left|\varphi_{a}^{\prime}(z)\right|^{2}\left(1-|z|^{2}\right) d m(z)
$$

is at most (6) by a conformal change of variable. Recalling that $\log ^{+} x \leq x$ for all positive $x$, we conclude

$$
\begin{aligned}
& \sup _{a \in \mathbf{D}} \sup _{0<r<1} \int_{0}^{2 \pi} \log ^{+}\left(\int_{0}^{r}\left|A\left(\varphi_{a}\left(s e^{i \theta}\right)\right)\right|\left|\varphi_{a}^{\prime}\left(s e^{i \theta}\right)\right|^{2}|f(a)|(1-s) d s\right) d \theta \\
& \lesssim \sup _{a \in \mathbf{D}} M_{\infty}(|a|, f) \int_{\mathbf{D}}|A(z)|\left(1-\left|\varphi_{a}(z)\right|^{2}\right) d m(z) .
\end{aligned}
$$

The quantity (13) is finite by (6) and the fact $f \in \mathcal{B}$. This proves (12), and hence Theorem 8.

\section{Proofs of Theorem 9 and Corollary 10}

The proof of Theorem 9 is based on the following result [21, Corollary 5.3]: If $f \in \mathcal{H}(\mathbf{D})$ and

$$
\int_{0}^{1} M_{\infty}\left(r, f^{\prime \prime}\right)^{2}\left(1-r^{2}\right)^{3} d r<\infty
$$


then $f \in \mathrm{VMOA}$.

Proof of Theorem 9. Let $f$ be a non-trivial solution of (1), and let $0<r_{0}<1$ be fixed. As in the proof of Theorem 1, we obtain

$$
M_{\infty}\left(r, f^{\prime \prime}\right) \leq M_{\infty}(r, A) M_{\infty}(r, f) \lesssim M_{\infty}(r, A) \exp \left(\int_{r_{0}}^{r} M_{\infty}(t, A)(1-t) d t\right)
$$

for $r_{0}<r<1$, where the comparison constant is independent of $r$. The assertion follows as $f \in \mathrm{VMOA}$ by (7) and (14).

Proof of Corollary 10. Fix any $0<r_{0}<1$. The coefficient condition (8) implies that there exists an absolute constant $0<C<\infty$ such that

$$
\exp \left(2 \int_{r_{0}}^{r} M_{\infty}(t, A)(1-t) d t\right) \lesssim\left(\log \log \frac{e}{1-r}\right)^{2 C}, \quad r_{0}<r<1,
$$

where the comparison constant is independent of $r$. The condition (7) is satisfied by a straight-forward computation, which completes the proof.

The following example shows that the coefficient condition (8) allows solutions of (1) to be unbounded.

Example 4. Let $0<\alpha<\infty$. Note that $f(z)=\left(\log \log \left(e^{e} /(1-z)\right)\right)^{\alpha}$ is a zerofree unbounded solution of (1) for

$$
A(z)=-\alpha \frac{\alpha-1+\left(\log \frac{e^{e}}{1-z}-1\right)\left(\log \log \frac{e^{e}}{1-z}\right)}{(1-z)^{2}\left(\log \frac{e^{e}}{1-z}\right)^{2}\left(\log \log \frac{e^{e}}{1-z}\right)^{2}}, \quad z \in \mathbf{D} .
$$

It is immediate that (8) is satisfied.

Acknowledgements. The author thanks the anonymous reviewer for valuable comments.

\section{References}

[1] Aleman, A.: Hilbert spaces of analytic functions between the Hardy and the Dirichlet space. - Proc. Amer. Math. Soc. 115:1, 1992, 97-104.

[2] Baernstein, A., II: Analytic functions of bounded mean oscillation. - In: Aspects of contemporary complex analysis (Proc. NATO Adv. Study Inst., Univ. Durham, Durham, 1979), Academic Press, London-New York, 1980, 3-36.

[3] Campbell, D., J. Cima, and K. Stephenson: A Bloch function in all $H^{p}$ classes, but not in BMOA. - Proc. Amer. Math. Soc. 78:2, 1980, 229-230.

[4] Cima, J. A.: The basic properties of Bloch functions. - Int. J. Math. Math. Sci. 2:3, 1979, $369-413$.

[5] Cima, J. A., and G. Schober: Analytic functions with bounded mean oscillation and logarithms of $H^{p}$ functions. - Math. Z. 151:3, 1976, 295-300.

[6] Frostman, O.: Potentiel d'équilibre et capacité des ensembles avec quelques applications á la theorie des fonctions. - Meddel. Lunds Univ. Mat. Sem. 3, 1935, 1-118.

[7] Gröhn, J., J.-M. Huusko, and J. RÄTTYÄ: Linear differential equations with slowly growing solutions. - Trans. Amer. Math. Soc. (to appear).

[8] Gröhn, J., A. Nicolau, and J. RÄTtyä: Mean growth and geometric zero distribution of solutions of linear differential equations. - J. Anal. Math. 134:2, 2018, 747-768.

[9] Gröhn, J., and J. RÄттуÄ: On oscillation of solutions of linear differential equations. - J. Geom. Anal. 27:1, 2017, 868-885. 
[10] Heittokangas, J.: A survey on Blaschke-oscillatory differential equations, with updates. In: Blaschke products and their applications, Fields Inst. Commun. 65, Springer, New York, 2013, 43-98.

[11] Heittokangas, J.: On complex differential equations in the unit disc. - Ann. Acad. Sci. Fenn. Math. Diss. 122, 2000, 1-54.

[12] Heittokangas, J., R. Korhonen, and J. RÄTtyä: Growth estimates for solutions of nonhomogeneous linear complex differential equations. - Ann. Acad. Sci. Fenn. Math. 34:1, 2009, $145-156$.

[13] Hille, E.: Remarks on a paper by Zeev Nehari. - Bull. Amer. Math. Soc. 55, 1949, 552-553.

[14] Huusko, J.-M., T. Korhonen, and A. Reijonen: Linear differential equations with solutions in the growth space $H_{\omega}^{\infty}$. - Ann. Acad. Sci. Fenn. Math. 41:1, 2016, 399-416.

[15] Laine, I.: Nevanlinna theory and complex differential equations. - Walter de Gruyter, Berlin, 1993.

[16] Nehari, Z.: The Schwarzian derivative and schlicht functions. - Bull. Amer. Math. Soc. 55, $1949,545-551$.

[17] Pau, J., and J. Á. PelÁEz: On the zeros of functions in Dirichlet-type spaces. - Trans. Amer. Math. Soc. 363:4, 2011, 1981-2002.

[18] Pommerenke, Ch.: On the mean growth of the solutions of complex linear differential equations in the disk. - Complex Variables Theory Appl. 1:1, 1982/83, 23-38.

[19] Pommerenke, Ch.: Schlichte Funktionen und analytische Funktionen von beschränkter mittlerer Oszillation. - Comment. Math. Helv. 52:4, 1977, 591-602.

[20] RÄттүё, J.: Linear differential equations with solutions in Hardy spaces. - Complex Var. Elliptic Equ. 52:9, 2007, 785-795.

[21] RÄтtYÄ, J.: $n$-th derivative characterisations, mean growth of derivatives and $F(p, q, s)$. Bull. Aust. Math. Soc. 68:3, 2003, 405-421.

[22] Ruscheweyh, St.: Two remarks on bounded analytic functions. - Serdica 11:2, 1985, 200-202.

[23] Schwarz, E.: Complex nonoscillation theorems and criteria of univalence. - Trans. Amer. Math. Soc. 80, 1955, 159-186.

[24] Steinmetz, N.: Normal families and linear differential equations. - J. Anal. Math. 117, 2012, $129-132$.

[25] Stephenson, K.: Functions which follow inner functions. - Illinois J. Math. 23:2, 1979, 259266.

[26] Zhaо, R.: On logarithmic Carleson measures. - Acta Sci. Math. (Szeged) 69:3-4, 2003, 605-618.

[27] ZHU, K.: Operator theory in function spaces. Second edition. - Math. Surveys Monogr. 138, Amer. Math. Soc., Providence, RI, 2007.

Received 1 August $2017 \bullet$ Accepted 8 December 2017 